\title{
Il n'y a pas le sujet: vive le citoyen!
}

\author{
RAUL ANTELO Universidade Federal de Santa Catarina, Brasil / antelo@floripa.com.br
}

\section{Resumen}

El sujeto, entendido como síntesis moderna de lo transcendental y lo fenoménico, se ha vuelto, en el pensamiento contemporáneo, un límite más que una fundación. La guerra civil o stasis es actualmente la auténtica condición de lo político y por ello la viabilidad del sujeto depende de su participación en la lucha democrática. Cincuenta años después de 1968, una exposición (iProteste! Resistencia en cartel) nos brinda no solo un atlas de memoria visual así como un llamado a la necesidad de resistencia sino también nos muestra el lugar del sujeto latinoamericano desde la perspectiva europea.

Palabras clave: sujeto / democracia / lucha política / resistencia / stasis

\section{There is no subject. Long live the citizen!}

\section{Abstract}

In contemporary thought, the subject, understood as a modern synthesis of the transcendental and the phenomenal, has become a limit, rather than a foundation. Civil war or stasis is nowadays the very condition of the political and that, as such, the viability of the subject depends on its participation in the agony of democracy. Fifty years after 1968, an exhibition (Protest! Resistance in the poster) provides not only an atlas of visual memory as well as an appeal to the need for resistance, but it also shows the place of the Latin-American subject from an European point of view.

Key words: subject / democracy / political struggle / resistance / stasis

Recibido: 20/02/2019. Aceptado: 25/03/2019

Para citar este artículo: Antelo, Raúl (2019). Il n'y a pas le sujet: vive le citoyen! El taco en la brea, 9 (diciembre-mayo), 57-63. Santa Fe, Argentina: UNL. DOI: 10.14409/tb.v1ig.8188 
Le narcissisme...! Il n'y a pas le narcissisme et le non-narcissisme; il y a des narcissismes plus ou moins compréhensifs, généreux, ouverts, étendus, et ce qu'on appelle le non-narcissisme n'est en général que l'économie d'un narcissisme beaucoup plus accueillant, hospitalier et ouvert à l'expérience de l'autre comme autre. Je crois que sans un mouvement de réappropriation narcissique, le rapport à l'autre serait absolument détruit, serait détruit d’avance. Il faut que le rapport à l'autre... -même s'il reste dissymétrique, ouvert sans réappropriation possible — il faut qu'il esquisse un mouvement de réappropriation dans l'image de soi-même pour que l'amour soit possible, par exemple. L'amour est narcissique. Alors, il y a des petits narcissismes, il y a des grands narcissismes, et il y a la mort au bout, qui est la limite. Dans l'expérience — si c'en est une— de la mort même, le narcissisme n'abdique pas absolument.(Derrida:212-213)

O livro que aqui nos reúne parece retomar a questão colocada por Joyce, um século atrás, no seu primeiro romance, Retrato do artista quando jovem (1916): «What was after the universe? Nothing. But was there anything round the universe to show where it stopped before the nothing place began?» (27). Potencialização da disseminação e desmaterialização da fronteira em benefício do limiar, a ideia tornava difícil o empreendimento de Nancy, Cadava e Connor. Por isso, na sua carta de fevereiro de 1986, convidando colegas a participarem do volume de Routledge que se impunha a tarefa de pensar o que vem após o sujeito, Jean-Luc Nancy colocava que

one of the major characteristics of contemporary thought is the putting into question of the instance of the «subject», according to the structure, the meaning, and the value subsumed under this term in modern thought, from Descartes to Hegel, if not to Husserl. The inaugurating decisions of contemporary thought whether they took place under the sign of a break with metaphysics and its poorly pitched questions, under the sign of a "deconstruction» of this metaphysics, under that of a transference of the thinking of Being to the thinking of life, or of the Other, or of language, etc., have all involved putting subjectivity on trial. A wide spread discourse of recent date proclaimed the subject's simple liquidation. Everything seems, however, to point to the necessity, not of a «return to the subject» (...), but on the contrary, of a move forward toward someone. (Cadava et al::5)

O sujeito, entendido ora como síntese entre o transcendental e o fenomênico, ora como alteridade integrada na identidade, ora como uma tecnologia disciplinar, tornou-se, contemporaneamente um limite, mais do que um limiar ou uma fundação, uma vez que ele é um valor artificial, contingente e efêmero. Vejamos o que vai de um momento a outro. Em sua contribuição ao volume de 1991, Alain Badiou concluía, baseado no peut-être mallarmaico, que «a subject is that which fends off the generic indiscernibility of a truth-a truth it effectuates in discernible finitude by an act of naming that leaves its referent in the future anterior of a condition». É esta marca, que diferencia duas disciplinas, a arqueologia («the future anterior of a condition») e a história, a responsável por um processo de nomeação.

A subject emptily names the universe yet-to-come that is obtained from the fact that an indiscernible truth supplements the situation. It is concurrently the finite real, the local stage of this supplementation. Naming is only empty insofar as it is pregnant with what its own possibility sketches out. A subject is the antonym of an empty idiom (langue). (Cadava et al::32) 
Se o sujeito é o antônimo do esquema abstrato e vazio da langue, ele configura a liberdade de um ato de fala. Em 1968, precisamente, Barthes defendia a ideia de que um texto é feito de escritas múltiplas, vindas de várias culturas e justapostas em constante diálogo, em paródia, em contestação; mas há um lugar em que essa multiplicidade se reúne, e esse lugar não é o do autor, mas o do leitor. A unidade de um texto não está portanto na sua origem, mas no seu destino, muito embora este destino já não possa ser pessoal: o leitor é um homem sem história, sem biografia, sem psicologia e se quisermos devolver à escrita o seu devir, é preciso inverter o seu mito: o nascimento do leitor tem de pagar-se com a morte do Autor. Ora, cinqüenta anos passados, o mesmo Alain Badiou, por ocasião do aniversário de 1968, perguntava-se o motivo da celebração dessa «morte do autor", encontrando duas respostas para tanto. Comemora-se o evento porque 68 , como o sujeito, ele está definitivamente morto; ou, pelo contrário, comemora-se porque é uma impugnação da ordem neoliberal, que daí em diante se impôs. Esta ambivalência se traduz ainda numa pluralidade interpretativa a respeito do evento e da multiplicidade de sujeitos aí envolvidos. Se for verdade então que o leitor que nasce em 68 é alguém sem história, sem biografia, nem psicologia, isto faz com que haja um 68 estudantil; um 68 operário; um 68 libertário, cujo pensador-fetiche é Guy Debord, e assim por diante. Mas a problematicidade desse evento é que 68 mesmo apresenta tanto uma linguagem comum compartilhada, quanto o esgotamento do uso dessa mesma linguagem.

Ce langage commun, ce marxisme ordinaire des partis et des syndicats «de lutte de classe», est en train de mourir dans les faits, car Mai 68 timidement, mais les années qui suivent vigoureusement, déploient une mise en cause massive de la légitimité des organisations historiques de la gauche, des syndicats, des partis, des leaders connus. Y compris dans les usines il y a contestation de la discipline, de la forme habituelle des grèves, de la hiérarchie du travail, de l'autorité syndicale sur les mouvements. L'action ouvrière et populaire est tirée hors de son cadre normal par des initiatives considérées comme anarchiques ou sauvages. Il y a enfin, et peut-être surtout, une critique radicale de la démocratie représentative, du cadre parlementaire et électoral, de la «démocratie» en son sens étatique, institutionnel, constitutionnel, auquel toutes les organisations «révolutionnaires» confient, de fait, leur destin, ne serait-ce que par leur adhésion invariable à ce que les révoltés du XIX $\mathrm{X}^{\mathrm{e}}$ siècle avaient très justement nommé le «crétinisme parlementaire».

Il ne faut surtout pas oublier (...) que le mot d’ordre final de Mai 68 est «Élections, piège à cons». Et ce n'est pas un simple emballement idéologique; des raisons précises justifient cette hostilité à la démocratie représentative. Après un grand mois de mobilisation étudiante, puis ouvrière et populaire sans précédent, le gouvernement réussit à organiser des élections et le résultat est la Chambre la plus réactionnaire que l'on ait connue! Il est alors clair pour tout le monde que le dispositif électoral n'est pas seulement, ni même principalement, un dispositif de représentation, c'est aussi un dispositif de répression des mouvements, des nouveautés, des ruptures.

À travers tout cela —outre cette "grande critique», pour parler comme les révolutionnaires chinois—, qui est essentiellement négatif, chemine une vision nouvelle, une vision de la politique qui tente de s'arracher à la vision classique. C'est cette tentative que j'appelle le quatrième Mai 68. Il cherche ce qui peut exister au-delà de la clôture du révolutionnarisme classique. Il cherche de manière aveugle, parce qu'il cherche en utilisant le même langage que celui qui domine dans la conception dont il veut se défaire. (Badiou:39-40) 
Ora, é nesse encaminhamento sagital, oblíquo, que Badiou ainda acha possível voltar ativamente a 68, porque «on a raison de se révolter». $E$, de fato, razões não faltam para tanto, se além do mais consideramos a relevância que o conceito de soulèvement, subsidiário de pathos, veio adquirindo no pensamento mais recente de Didi-Huberman, por exemplo (2013, 2016, 2017a, 2017b). Ele é quase a inversão da sobrevivência, se lembramos, justamente, de uma das palavras de ordem de 68: «il n'y a que la Révolution qui puisse nous délivrer de l'ignobile poids mort des survivances».

Com efeito, a partir do alastramento do capitalismo concentracionário e o consequente enfraquecimento dos Estados nacionais, a situação pós-68 cria uma relação sistêmica entre terrorismo e Estado de segurança, em que o próprio Estado precisa do medo para se legitimar, e nesse sentido, ele mesmo cria o terror ou, quando muito, não impede que ele esteja no comando das iniciativas. E por terror temos que entender a flexibilização trabalhista, o fim do Estado de Bem-estar social, a reforma previdenciária etc. Isto acarreta uma mudança do estatuto político dos sujeitos, os cidadãos, aos quais se atribuía, em fase anterior, a titularidade da soberania. No Estado de segurança, porém, constatamos uma despolitização progressiva dos sujeitos, cuja participação na vida política se reduz simplesmente a pesquisas de opinião e sondagens eleitorais. A sublevação, a revolta apregoada por Badiou ou Didi-Huberman, é portanto uma forma de tornar político esse elemento impolítico, que atravessa toda a cena pública no século XXI. Afinal, se «Je est un autre», se o Eu vem de um Outro, a constituição do sujeito só pode ser paranoica. Daí o método daliniano: a paranoia-crítica.

Qual é, a rigor, o início desse processo? Sabemos que, concluída a guerra, Georges Bataille pretendia lançar pelas Èditions du Minuit uma coleção de ensaios sobre o uso das riquezas, à qual não seriam alheias de certo as contribuições de Roger Caillois, analisando dados latino-americanos. Num panfleto de propaganda, escrevia então Bataille (1948):

La terre est le lieu de mouvements d'énergie qui s'ordonnent selon les lois générales: en particulier, les hommes ont besoin de sommes d'énergies considérables afin de vivre et de croître, mais l'attention n'a pas suffisamment porté sur ce fait: l'énergie dont ils vivent sert sans cesse à la production d'énergie nouvelle, mais les hommes produisent toujours plus d'énergie qu'ils n'en utilisent. Il y a un continuel excédent qu'épuisent aussi bien le luxe, le loisir ou les guerres. L'usage de cet excédent, l'«usage des richesses» pose ainsi des problèmes aigus. À chaque type de civilisation correspondent des usages différents. Chaque moment historique introduit des crises. Un choix impliquant des risques toujours offert aux sociétés: que doivent-elles faire de l'excédent dont elles disposent? Elles peuvent le consumer dans des fêtes. Elles peuvent l'utiliser à leur croissance (tant à la croissance démographique qu'à celle des forces de production). Elles peuvent aussi méconnaître la nécessité où elles sont de choisir en tenant compte des possibilités données: c'est peut-être le cas actuel. Dès lors, un désordre insensé s'empare de la terre, l'énergie multipliée entretient un mouvement de catastrophe. (1948)

E, paralelamente, Caillois retomava a análise bataillana do Tibete ${ }^{1}$ e suas próprias pesquisas sobre o jogo do bicho no Brasil (a economia para-estatal) para assim concluir:

Déjà Ruskin le remarquait avec force, écrivant : «J'ai intrépidement déclaré que votre prétendue science de l'Économie politique n'en est pas une, parce que justement elle a omis la branche la plus importante de la question: la dépense». Cet avertissement a d'ailleurs été partiellement entendu. Toutefois il ne semblera 
pas inutile de souligner encore que s'interroger sur les buts de la dépense plutôt que sur les procédés de l'acquisition, c'est pour une part se dégager des préjugés de l'époque, d’autant plus dangereux qu'ils sont insensibles et que c'est s'engager soi-même à vraiment réfléchir aux fins dernières de la civilisation. (436)

Ora, a leitura neoliberal chamaria, anos depois, essa desordem esbanjadora de populismo. Ele gasta mais do que arrecada e acumula, daí a necessidade de constantemente ajustar a economia a padrões de eficiência anti-assistencialista, que não poupa, entretanto, em investimentos de defesa ou pesquisa armamentista. Esse Estado de segurança, assentado em capitalismo financeiro e concentracionário, funciona, na prática, como um Estado policialesco, em que o eclipse do poder judiciário generaliza o arbítrio discricional do controle, ao passo que sua aliança com a sociedade do espetáculo, através da mídia, igualmente oligopólica, cria, ao mesmo tempo, a ilusão de normalidade, através de um estado de emergência, que, mesmo sem credenciais, comporta-se cada vez mais como soberano inconteste. Através da despolitização progressiva dos sujeitos, ora tornados eles mesmos terroristas potenciais, o Estado abandona assim a política para se instalar em espaço indecidível, onde o público e o privado constantemente se embaralham, criando inúmeras aporias para o direito. A disseminação de técnicas de gestão, dispositivos permanentes de avaliação, coaches e conselheiros são o suplemento social do sujeito neoliberal, que vivendo para além do próprio limite, no gozo empreendedor, vive ameaçado pelo fracasso (o stress, o ataque de pânico, a depressão) que nada mais são do que o próprio desconhecimento de si.

Um economista neoliberal próximo de Reagan, com quem aliás colaborou, George Gilder, caracteriza a transformação contemporânea como a ilustração de um princípio econômico clássico conhecido como lei de Jean-Baptiste Say, segundo a qual a oferta cria a demanda. Nessa forma contemporânea do potlatch, residiria tanto a manutenção quanto o ultrapassamento da situação moderna, pessoal e subjetiva, que o levam até mesmo a predizer a existência de um componente teológico no capitalismo, retomando, com sentido inverso, a tese benjaminiana de 1921. Mas há aqui um debate potencial. São os dispositivos completamente poderosos e bem sucedidos no sentido de construírem uma subjetividade? É suficiente a desconstrução da democracia para gerar um sujeito apático? O sujeito é, como pensam alguns herdeiros de Bataille, como os foucaultianos, apenas uma produção histórica regulada pelos dispositivos de poder e saber? Ou, pelo contrário, na linha de Freud, Heidegger, Lacan ou Badiou, caberia pensar que ainda existem elementos, na própria estruturação do sujeito, que nenhuma ordem político-histórica consegue integrar de forma total e acabada?

No outono de 1941, quando Newton Freitas morava em Buenos Aires, envia a Mário de Andrade a tradução de The Wild Palms de Faulkner, feita por Borges. Mário nunca encontrou, nos quatro anos de vida que lhe restavam, tempo para sequer abrir o exemplar. Ficou sem conhecer Faulkner. Quase vinte anos depois, Michel, o protagonista cafajeste de Acossado, de Godard (1960), é uma sutil reencarnação da apatia de Mário. Enquanto ele, fissurado, quer logo se deitar com a protagonista enquanto ela resiste, há um diálogo muito esclarecedor entre Michel e Patricia:

Patricia: - Tu connais William Faulkner?

Michel: -Non..., qui est-ce? Tu as couché avec lui?

Patricia: - Mais non, mon coco.

Michel: - Alors je me fous de lui... Enlève ton Jersey. 
Patricia: - C'est un romancier que j'aime bien. Tu as lu Les palmes sauvages?

Michel: - Je te dis que non... Enlève ton chandail.

Patricia: -Ecoute. La dernière phrase, c'est très beau: «Between grief and nothing, I will take grief». Entre le chagrin et le néant, je choisis le chagrin... Et toi, tu choisirais quoi?

Michel: - (...) Le chagrin, c'est idiot. Je choisis le néant. C'est pas mieux, mais le chagrin, c'est un compromis. Faut tout ou rien. Puis maintenant, je le sais...

Michel, adiantando-se ao sentimento 68, escolhe o néant, o niilismo. È um horizonte do que vem após o sujeito. Nunca the grief, le chagrin, o luto. Ou seja, o modernismo que elabora o luto do nacional e do popular. Antes, o nada, a liberdade.

Uma exposição contemporânea, também suscitada pelo cinqüentenário de 68 , pode ainda nos auxiliar para ilustrar os limites dessa questão do sujeito e sua vocação de liberdade. Protest Eine Zukunftspraxis! (Proteste. A Estética da Resistência) esteve em cartaz no Museum für Gestaltung Zurich, entre abril e setembro de 2018. Nela houve cartazes de James Victore, David Tartakover, Vincent Perrottet, Tomi Ungerer, Klaus Staeck, John Heartfield e da Asamblea de los Artistas Revolucionarios de Oaxaca (ASARO), entre outros. O diretor do Cabaret Voltaire, Adrian Notz, apresentou «We», um manifesto (2018), que, a rigor, é uma proliferação de reivindicações onde a subjetividade, mesmo plural, não deixava de ocupar o centro da cena. E, quanto às imagens e revoltas, além da presença, como um todo, bastante marginal da América Latina, era surpreendente ver que as manifestações escolhidas obedeciam, prioritariamente, ao modelo da representação democrático-partidária, liderada pelo Partido Comunista, algo que, sabemos, está longe de ser hegemônico no Continente. Estavam assim presentes, entre outras, imagens do Black Power, das lutas do Oriente próximo, da Síria e da Ucrânia, o feminismo, o Fórum econômico mundial (WEF), a guerra civil espanhola, Vietnã, a guerra fria e a da Iugoslávia, a guerra do Golfo, a descolonização, as migrações, os movimentos anti-apartheid ou, mais amplamente, as lutas pelos direitos civis nos EEUU, o pacifismo, Occupy ou o efeito estufa. A América Latina comparecia exclusivamente pelos movimentos de libertação. «Garantida pela guerrilha, explicava o folder de mão, a vitória da revolução cubana abre a via aos movimentos de libertação em toda a América Latina. Combatem os regimes militares e prometem uma alternativa ao socialismo real. As comunidades eclesiais de base sustentam seu esforço». Em compensação, as manifestações de dissidência ou aberta rebelião motorizadas por movimentos populistas, antes ou depois de 1959, eram pouco menos que solapadas ou obliteradas. ${ }^{2}$ Em suma, estereotipia eurocêntrica com relação aos processos emancipatórios latino-americanos e, paralelamente, reforço da subjetividade de autor como agente exclusivo das transformações. Em uma palavra, a equação passava olímpicamente ao largo da condição colonial e de problemáticas a ela ligadas, tais como a questão étnica ou de gênero. Ou seja, negligenciava o com de todo ser-com, de toda comunidade, algo que se tornaria um autêntico desafio na Europa atual, a presença daqueles sujeitos chamados, estrangeiros residentes (Di Cesare). Relembremos, para concluir, a ponderação de Nancy:

Un sujet unique ne pourrait même pas se désigner et se rapporter à soi comme sujet. Un sujet, au sens le plus classique du terme, ne suppose pas seulement sa propre distinction d'avec l'objet de sa représentation ou de sa maîtrise: il suppose au moins tout autant sa propre distinction d'avec d'autres sujets dont il puisse distinguer l'ipséité (voire, si on veut le dire ainsi, l'aséité) d'avec son propre foyer de représentation ou de maîtrise. L'avec est donc la supposition du «soi» en général. 
Mais il n'est précisément plus une supposition sous-jacente, sur le mode de l'auto-présupposition infinie de la sub-stance sub-jective. Comme sa fonction syntaxique l'indique, «avec» est la pré-position de la position en général, et il fait ainsi sa disposition. (Nancy:60-61)

\section{Notas}

1 «Toujours, dans l'ensemble, une société produit plus qu'il n'est nécessaire à sa subsistance; elle dispose toujours d'un surplus. Et c'est précisément l'usage qu'elle en fait qui la détermine. Le surplus est la cause de l'agitation, des changements de

structure et de toute l'histoire» (Bataille 1947).

2 Baste relembrar que a queda do peronismo, em 1955, prevista aliás por Caillois, leva a Argentina a contrair o primeiro empréstimo com o FMI.

\section{Referências bibliográficas}

Badiou, A. (2018). On a raison de se révolter. L'actualité de Mai 68. Paris: Fayard.

Bataille, G. (1947). Le paradoxe du Thibet. Critique, (12), 427-438.

(1948). Boletim de publicidade das Éditions de Minuit.

Beauregard, G. (Produtor), Godard, J.-L. (1960). À bout de souffle (título português Acossado). França: Les Films Impéria, Les Productions Georges de Beauregard, Société Nouvelle de Cinématographie.

Cadava, E., Connor, P., Nancy, J.-L. (1991). Who comes after the subject? New York: Routledge.

Caillois, R. (1948). Économie quotidienne et jeux de hasard en Amérique ibérique. Annales. Economies, sociétés, civilisations, a. 3, n 4, 427-436. [Com o título proposto por Bataille, «L'usage des richesses», 0 texto se incorporaria, em 1964, a seu volume Instincts et société. Essais de sociologie contemporaine.].

Derrida, J. (1992). Points de suspension. Paris: Galilée.

Di Cesare, D. (2018). Stranieri residenti. Una filosofia della migrazione. Torino: Bollatti Boringhieri.

Didi-Huberman, G. (2013). Soulèvements poétiques (poésie, savoir, imagination). Po\&sie, (143), 153-157. et al. (2016). Soulèvements. Catalogue de l'exposition (2016-2017) au Jeu de Paume, Concorde. Pref. Marta Gili. Collab. Nicole Brenez, Judith Butler, Georges Didi-Huberman, Marie-José Mondzain, Antonio Negri et Jacques Rancière. Paris: Jeu de Paume/Gallimard. (2017a). Levantes. São Paulo: SESC. Tradução de Edgard de Assis Carvalho et al. (2017b). Sublevaciones. Buenos Aires: MUNTREF.

Joyce, J. (1916). A Portrait of the Artist as a Young Man. Ed. Hans Walter Gabler et al. New York: Vintage.

Nancy, J.-L. (1996). Être singulier-pluriel. París: Galilée. 